\title{
Universität des Saarlandes
}

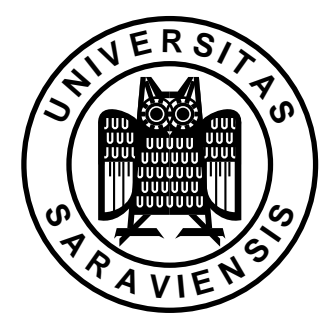

Fachrichtung 6.1 - Mathematik

\author{
Preprint Nr. 197
}

A General Structure Tensor Concept and Coherence-Enhancing Diffusion Filtering for Matrix Fields

\author{
Bernhard Burgeth, Stephan Didas and \\ Joachim Weickert
}

Saarbrücken 2007 



\title{
A General Structure Tensor Concept and Coherence-Enhancing Diffusion Filtering for Matrix Fields
}

\author{
Bernhard Burgeth \\ Saarland University \\ Department of Mathematics \\ P.O. Box 151150 \\ 66041 Saarbrücken \\ Germany \\ burgeth@mia.uni-saarland.de \\ Stephan Didas \\ Saarland University \\ Department of Mathematics \\ P.O. Box 151150 \\ 66041 Saarbrücken \\ Germany \\ didas@mia.uni-saarland.de \\ Joachim Weickert \\ Saarland University \\ Department of Mathematics \\ P.O. Box 151150 \\ 66041 Saarbrücken \\ Germany \\ weickert@mia.uni-saarland.de
}


Edited by

FR 6.1 - Mathematik

Universität des Saarlandes

Postfach 151150

66041 Saarbrücken

Germany

Fax: $\quad+496813024443$

e-Mail: preprint@math.uni-sb.de

WWW: http://www.math.uni-sb.de/ 


\begin{abstract}
Coherence-enhancing diffusion filtering is a striking application of the structure tensor concept in image processing. The technique deals with the problem of completion of interrupted lines and enhancement of flow-like features in images. The completion of line-like structures is also a major concern in diffusion tensor magnetic resonance imaging (DT-MRI). This medical image acquisition technique outputs a $3 \mathrm{D}$ matrix field of symmetric $3 \times 3$-matrices, and it helps to visualise, for example, the nerve fibers in brain tissue. As any physical measurement DT-MRI is subjected to errors causing faulty representations of the tissue corrupted by noise and with visually interrupted lines or fibers.

In this paper we address that problem by proposing a coherenceenhancing diffusion filtering methodology for matrix fields. The approach is based on a generic structure tensor concept for matrix fields that relies on the operator-algebraic properties of symmetric matrices, rather than their channel-wise treatment of earlier proposals.

Numerical experiments with artificial and real DT-MRI data confirm the gap-closing and flow-enhancing qualities of the technique presented.
\end{abstract}

Keywords: matrix field, symmetric matrix, diffusion tensor MRI, coherenceenhancing diffusion filtering, CED, structure tensor

\title{
1 Introduction
}

Coherence-enhancing diffusion (CED) filtering has been introduced in [25, 26] as an image restoration technique that enhances flow-like structures in scalar and vector-valued images. It regularises images polluted by noise, and it is capable of closing gaps in line-like structures. CED-filtering of a scalar image $f$ defined on the image domain $\Omega \subset \mathbb{R}^{d}$ produces simplified versions $u(\cdot, t)$ of $f$ as solutions of the partial differential equation (PDE)

$$
\begin{array}{rlrl}
\partial_{t} u-\operatorname{div}(D \cdot \nabla u) & =0 & & \text { in } I \times \Omega, \\
\partial_{n} u & =0 & \text { in } I \times \partial \Omega, \\
u(x, 0) & =f(x) & \text { in } \Omega,
\end{array}
$$

where $I=\left[0, T\left[\right.\right.$ is a potentially unbounded time interval, and $\partial_{n} u$ denotes the outer normal derivative of $u$ at the boundary $I \times \partial \Omega$.

In view of the qualities mentioned it would be desirable to have this method at our disposal for matrix-valued images, or matrix fields for short. For 
example, such a filter could serve as a pre-processing step for the so-called fiber tracking in diffusion tensor magnetic resonance imaging (DT-MRI); see [28], chapter 6 by Cook et al. and chapter 7 by Yushkevich et al. as well as the literature cited therein. In parts of the brain, e.g. the corpus callosum, nerve fibers form bundles with a coherent structure. In principle the matrix field produced by DT-MRI allows for the application of fiber tracking techniques and hence for the accurate visualisation of the nerve fibers. However, ubiquitous measurement errors during acquisition cause gaps and interruptions in the fibers' visualisation. Therefore a fiber tracking techniques would benefit from a prior filtering by a matrix-valued counterpart of CED. It is the goal of this chapter to extend CED and the underlying structure tensor concept to matrix-valued images.

Promising proposals to generalise nonlinear regularisation methods and related diffusion filters for scalar images to matrix fields have been made in $[7,8]$. These approaches are based on a basic differential calculus for matrix fields. These concepts had direct implications for chapter 18 by Steidl et al., chapter 19 by Lie et al., and they will be useful in the context of this chapter as well. Edge-enhancing diffusion, EED, a concept related to CED has been extended to the setting of matrix fields in [4]. Other PDE-based methods for the regularisation of matrix fields have been proposed, for example, in $[11,22,23,27,10,19,13]$. Approaches to tensor field regularisation with a more differential geometric background are explored in $[20,18,2]$ where the set of positive definite matrices is endowed with a Riemannian metric stemming from the DT-MRI field. Diffusion over tensor fields based on Lie groups is considered in [15] and in chapter 17 by Y. Gur and N. Sochen.

The essential ingredient in equation (1) underlying CED is the diffusion tensor $D$ of the scalar image $u$ which steers the diffusion process: It amplifies diffusion along flow-like structures, and hinders diffusion perpendicular to those pattern. Postponing the detailed construction of $D$ to the subsequent Section 2, for now we only remark that it is a function of the structure tensor [14] which is given by

$$
S_{\rho}(u(x)):=G_{\rho} *\left(\nabla u(x) \cdot(\nabla u(x))^{\top}\right)=\left(G_{\rho} *\left(\partial_{x_{i}} u(x) \cdot \partial_{x_{j}} u(x)\right)\right)_{i, j=1, \ldots, d} .
$$

Here $G_{\rho} *$ indicates a convolution with a Gaussian of standard deviation $\rho$, however, more general averaging procedures can be used. If $\nabla u(x) \neq 0$ the matrix

$$
\left(\nabla u(x) \cdot(\nabla u(x))^{\top}\right)
$$

has rank one, the eigenvector $\nabla u(x)$ belongs to the only non-zero eigenvalue $|\nabla u(x)|^{2}$. The eigenvalues represent the contrast in the directions of the 
eigenspaces. The averaging process then creates a matrix with full rank which contains valuable directional information. Note that the averaging of the structure tensor avoids cancellation of directional information. If one would average the gradients instead, neutralisation of vectors with opposite sign would occur. In many applications it is advantageous to use a presmoothed image $u_{\sigma}:=G_{\sigma} * u$ instead of $u$ in order to reduce the influence of noise for better numerical results. The structure tensor is a classical tool in image processing to extract directional information from an image going back to [17], for more details the reader is referred to [3] and the literature cited there.

It is not straightforward to generalise both the structure and the diffusion tensor concept to the setting of matrix-valued images.

To fix notation in this work matrix-valued images or matrix fields $M(x)$ are considered as mappings from $\mathbb{R}^{d}$ into the set $\operatorname{Sym}_{n}(\mathbb{R})$ of symmetric $n \times n$ matrices

$$
M: x \mapsto M=\left(m_{i, j}(x)\right)_{i, j=1, \ldots, n} \in \operatorname{Sym}_{n}(\mathbb{R}) .
$$

and denoted by capital letters while indexed lower case letters indicate their components.

Di Zenzo's approach [12] to build a structure tensor for multi-channel images has been generalised in a straight forward manner as follows: Each channel considered as independent scalar image gives rise to a structure tensor, then these structure tensors are summed up to give a standard structure tensor $[27,5]$ :

$$
J_{\rho}(U(x)):=\sum_{i, j=1}^{n} S_{\rho}\left(u_{i, j}(x)\right) .
$$

This construction has been refined to a customisable structure tensor in [21]. There the resulting structure tensor is a weighted sum of tensors of scalar quantities that are now not just the channels, but other meaningful scalar quantities derived from the matrix field. The weights are provided by the user, and depending on the choice of weights the emerging structure tensor has a sensitivity for certain features of the matrix field. A special constellation of the weights turns the customisable structure tensor into the standard structure tensor from $[27,5]$. It is important to mention that in case of a $3 \mathrm{D}$ matrix field of $3 \times 3$ symmetric matrices these concepts yield also a $3 \times 3$ structure tensor, the very same order as a 3D scalar image.

Here we opt for a different approach: We assume an operator-algebraic view on symmetric matrices as finite dimensional instances of selfadjoint Hilbert space operators. The exploitation of the algebraic properties of matrices, ensures proper interaction between the different matrix channels. This is a 
decisive advantage over the standard component-wise treatment of vectorvalued images.

In this chapter we will present a general concept for a large size, second order structure tensor, a $n d \times n d$-matrix, that carries all the directional information of the matrix field. We will show how this information can be deduced from this full tensor by a reduction process. This reduction process illuminates also its connection to the structure tensors mentioned above.

The chapter is structured as follows: The next Section 2 is devoted to a brief review of coherence enhancing diffusion filtering of scalar images. Notions necessary to construct the diffusion tensor and a basic differential calculus for matrix fields necessary to construct the diffusion tensor is provided in Section 3. In Section 4 we propose a novel structure tensor concept for matrix fields, study some of its properties by investigating the connection to already known structure tensors for matrix-valued data. We then feature the potential of this concept by proposing a coherence enhancing diffusion for matrix fields in Section 5. We report on the results of our experiments with matrix-valued coherence enhancing diffusion applied to synthetic data and real DT-MRI images in Section 6. Section 7 is made up by concluding remarks.

\section{Synopsis of Coherence Enhancing Diffusion}

The rationale behind the construction of the diffusion tensor $D$ for grey value images as proposed in [25] is as follows: The matrix $S_{\rho}(u)$ as the positive average of different symmetric positive semidefinite matrices has the very same property. Hence $S_{\rho}(u)$ has an orthonormal system $\left\{w_{1}, \ldots, w_{d}\right\}$ of eigenvectors corresponding to the non-negative eigenvalues $\mu_{1} \geq \mu_{2} \geq \ldots \geq$ $\mu_{d} \geq 0$ indicating the contrast in each direction. In the line defined by $w_{d}$, the coherence direction the contrast is the least compared to other orientations, since $w_{d}$ belongs to the smallest eigenvalue $\mu_{d}$. The coherence or anisotropy of an image structure essentially is captured in eigenvalue distribution of the structure tensor $S_{\rho}$. In [25] the quantity

$$
\kappa:=\sum_{i=1}^{d-1} \sum_{j=i+1}^{d}\left(\mu_{i}-\mu_{j}\right)^{2}
$$

is proposed to measure coherence. Strongly differing eigenvalues result in a large value of $\kappa$, while similar values produce a small $\kappa$-value indicating a structure with isotropic character. The matrix $D$ has the same eigenvectors as $S_{\rho}$, however, its eigenvectors $\lambda_{i}$ are altered via the tensor transfer map $H$ according to

$$
\lambda_{i}:=H\left(\mu_{i}\right):=\alpha \quad \text { for } i=1, \ldots, d-1
$$


and

$$
\lambda_{d}:=H\left(\mu_{d}\right):= \begin{cases}\alpha & \text { if } \kappa=0, \\ \alpha+(1-\alpha) \exp \left(-\frac{C}{\kappa}\right) & \text { else. }\end{cases}
$$

with a threshold $C>0$. With this choice of $D$ the CED-filter (1) smoothes mainly along the coherence direction $w_{d}$ with a diffusivity $\lambda_{d}$ that increases with $\kappa$. Note that $\min \left\{\lambda_{i}, i=1, \ldots, d\right\}=\alpha>0$, which causes $D$ to be uniformly positive definite and enforces a diffusion no matter how isotropic $(\kappa \downarrow 0)$ the image structure becomes.

It is our task in this chapter to extend the notion of structure and diffusion tensor from scalar images to matrix fields. Ultimately we aim at coherence enhancing diffusion filtering of matrix fields. To do so we have to clarify what is meant by the partial derivative, the gradient and, most of all, the suitable structure tensor of a matrix field. This is done in the next section.

\section{Basic Differential Calculus for Matrix Fields}

In this section we provide briefly the basic definitions for the formulation of a differential calculus for matrix fields. This material is instigated in [6] but for a more detailed exposition the reader is referred to [7].

1. Functions of matrices. The standard definition of a function $h$ on $\operatorname{Sym}_{n}(\mathbb{R})$ is given by $[16]$ :

$$
h(U)=V^{\top} \operatorname{diag}\left(h\left(\lambda_{1}\right), \ldots, h\left(\lambda_{n}\right)\right) V \in \operatorname{Sym}_{n}(\mathbb{R}),
$$

if $U=V^{\top} \operatorname{diag}\left(\lambda_{1}, \ldots, \lambda_{n}\right) V$ is the spectral- / eigendecomposition of the symmetric matrix $U$, and if $\lambda_{1}, \ldots, \lambda_{n}$ lie in the domain of definition of $h$. We encountered already an example of a function of a symmetric matrix; the diffusion tensor as a function of the structure tensor $S_{\rho}$ with coherence $\kappa$ under the tensor transfer map $H, D=H\left(S_{\rho}\right)$.

2. Partial derivatives. Let $\omega \in\left\{x_{1}, \ldots, x_{d}, t\right\}$ denote a spatial or temporal variable, and set $(x, t)=\left(x_{1}, \ldots, x_{d}, t\right)$. The partial derivative for matrix fields is naturally defined component-wise as the limit of a difference quotient:

$$
\begin{aligned}
\bar{\partial}_{\omega} U(x, t) & =\lim _{h \rightarrow 0} \frac{U\left((x, t)+h \cdot e_{k}\right)-U(x, t)}{h} \\
& =\left(\lim _{h \rightarrow 0} \frac{u_{i j}\left((x, t)+h \cdot e_{k}\right)-u_{i j}(x, t)}{h}\right)_{i, j} \\
& =\left(\partial_{\omega} u_{i j}(x, t)\right)_{i, j}
\end{aligned}
$$


where $e_{k}:=(0, \ldots, 0,1,0, \ldots, 0) \in \mathbb{R}^{d+1}$ denotes the $k$ th unit vector of space-time $\mathbb{R}^{d+1}$. The generalisation to directional derivatives is straight forward. In this case $\omega$ would denote an appropriate unit vector. Higher order partial differential operators, such as the Laplacian or other more sophisticated operators, find their natural counterparts in the matrix-valued framework in this way as well. It is worth mentioning that for the operators $\bar{\partial}_{\omega}$ a product rule holds:

$$
\left.\bar{\partial}_{\omega}(A(x) \cdot B(x))=\left(\bar{\partial}_{\omega} A(x)\right) \cdot B(x)\right)+A(x) \cdot\left(\bar{\partial}_{\omega} B(x)\right) .
$$

3. Generalized gradient of a matrix field. The gradient of a matrix field with sufficiently smooth component functions is defined via

$$
\bar{\nabla} U(x):=\left(\bar{\partial}_{x_{1}} U(x), \ldots, \bar{\partial}_{x_{d}} U(x)\right)^{\top} \in\left(\operatorname{Sym}_{n}(\mathbb{R})\right)^{d}
$$

Hence, the generalised gradient $\bar{\nabla} U(x)$ at a voxel $x$ is regarded as an element of the module $\left(\operatorname{Sym}_{n}(\mathbb{R})\right)^{d}$ over $\operatorname{Sym}_{n}(\mathbb{R})$ in close analogy to the scalar setting where $\nabla u(x) \in \mathbb{R}^{d}$. Hence, in the sequel we will call a mapping from $R^{d}$ into the module $\left(\operatorname{Sym}_{n}(\mathbb{R})\right)^{d}$ a module field rather than a vector field, the later one being a mapping from $R^{d}$ into a vector space.

Note that this definition of a generalised gradient is different from one that might be expected when viewing a matrix as a tensor (of second order). According to differential geometry concepts their derivatives are tensors of third order. However, we adopt an operator-algebraic point of view: The matrices are self-adjoint operators that can be added, multiplied with a scalar, and concatenated. Thus, they form an algebra, and we aim at consequently replacing the field $\mathbb{R}$ by the algebra $\operatorname{Sym}_{n}(\mathbb{R})$ in the scalar, that is, $\mathbb{R}$-based formulation of differential calculus.

4. For the sake of completeness we include the formal definition of the generalized structure tensor of a matrix field here. We will discuss its derivation, properties and application in the next section. The novel structure tensor for a matrix field is given by

$$
\begin{aligned}
\overline{\mathcal{S}}_{L}(U(x)) & :=G_{\rho} *\left(\bar{\nabla} U(x) \cdot(\bar{\nabla} U(x))^{\top}\right) \\
& =\left(G_{\rho} *\left(\bar{\partial}_{x_{i}} U(x) \cdot \bar{\partial}_{x_{j}} U(x)\right)\right)_{i, j=1, \ldots, d} .
\end{aligned}
$$

5. Symmetrised product of symmetric matrices. The product of two symmetric matrices $A, B \in \operatorname{Sym}_{n}(\mathbb{R})$ is not symmetric unless the 
matrices commute. However, it is vital to our interests to have a symmetrised matrix product at our disposal. There are numerous options to define a symmetrised matrix product, however, we concentrate on a specific one known from algebra and called Jordan product:

$$
A \bullet_{J} B=\frac{1}{2}(A B+B A) \quad \text { for } A, B \in \operatorname{Sym}_{n}(\mathbb{R}) .
$$

For commuting $A$ and $B$ we have $A \bullet_{J} B=A \cdot B$. This product is commutative and distributive but not associative. It is one half of the anti-commutator of $A$ and $B$, but due to its additive structure no determinant product rule holds. Most important, it does not preserve the positive semidefinitness of its arguments as the following simple example shows:

$$
\left(\begin{array}{ll}
2 & 0 \\
0 & 0
\end{array}\right) \bullet_{J}\left(\begin{array}{ll}
1 & 1 \\
1 & 1
\end{array}\right)=\frac{1}{2}\left(\left(\begin{array}{ll}
2 & 2 \\
0 & 0
\end{array}\right)+\left(\begin{array}{ll}
2 & 0 \\
2 & 0
\end{array}\right)\right)=\left(\begin{array}{ll}
2 & 1 \\
1 & 0
\end{array}\right)
$$

with

$$
\operatorname{det}\left(\begin{array}{ll}
2 & 1 \\
1 & 0
\end{array}\right)=-1
$$

Hence, simply multiplying each matrix in a DT-MRI-field by a positive definite matrix employing the Jordan product might produce a matrix field that cannot be interpreted anymore as DT-MRI data. This is not desirable.

\section{Remarks:}

1) It should be mentioned that the logarithmic multiplication introduced in [1] and given by $A \bullet_{L} B:=\exp (\log (A)+\log (B))$ is defined only for positive definite matrices. However, the matrix-valued diffusion proposed here requires the multiplication to be able to cope with the factor matrices being indefinite. Furthermore matrix fields that are not necessarily positive semidefinite should also be within the reach of our PDE-based filtering. Hence the logarithmic multiplication is not suitable for our purpose.

2) The proposed notions for a calculus on symmetric matrix fields are extensions of the calculus of scalar multivariate functions. As such it must be possible to regain the scalar calculus from the newly introduced matrixvalued framework by specification. There are two ways to view scalar calculus as a special case of the matrix calculus: Clearly, setting $n=1$ turns the matrix field into a scalar function. However, one can also embed the set of real numbers $\mathbb{R}$ into the set of symmetric matrices $\operatorname{Sym}_{n}(\mathbb{R})$ by the identification $\mathbb{R} \ni r \longleftrightarrow r \cdot I_{n}$ with the $n \times n$ identity matrix $I_{n}$. Hence, aside from having a certain simplicity, it is mandatory that the proposed extensions collapse 
to the scalar calculus when making the transition from scalar functions to matrix fields in one way or the other.

We summarised the definitions from above and juxtapose them with their scalar counterparts in the subsequent table 1 . The matrix field $U(x)$ is assumed to be diagonisable with $U=\left(u_{i j}\right)_{i j}=V^{\top} \operatorname{diag}\left(\lambda_{1}, \ldots, \lambda_{n}\right) V$, where $V \in O(n)$, the set of all orthogonal $n \times n$-matrices, and $\lambda_{1}, \ldots, \lambda_{n} \in \mathbb{R}$.

\begin{tabular}{|c|c|c|}
\hline Setting & scalar valued & matrix-valued \\
\hline function & $h:\left\{\begin{array}{l}\mathbb{R} \longrightarrow \mathbb{R} \\
x \mapsto h(x)\end{array}\right.$ & $h:\left\{\begin{array}{l}\operatorname{Sym}_{n}(\mathbb{R}) \longrightarrow \operatorname{Sym}_{n}(\mathbb{R}) \\
U \mapsto V^{\top} \operatorname{diag}\left(h\left(\lambda_{1}\right), \ldots, h\left(\lambda_{n}\right)\right) V\end{array}\right.$ \\
\hline $\begin{array}{c}\text { partial } \\
\text { derivatives }\end{array}$ & $\begin{array}{c}\partial_{\omega} u \\
\omega \in\left\{t, x_{1}, \ldots, x_{d}\right\}\end{array}$ & $\begin{array}{l}\bar{\partial}_{\omega} U:=\left(\partial_{\omega} u_{i j}\right)_{i j} \\
\omega \in\left\{t, x_{1}, \ldots, x_{d}\right\}\end{array}$ \\
\hline gradient & $\begin{array}{c}\nabla u(x):=\left(\partial_{x_{1}} u(x), \ldots, \partial_{x_{d}} u(x)\right)^{\top} \\
\nabla u(x) \in \mathbb{R}^{d}\end{array}$ & $\begin{aligned} \bar{\nabla} U(x) & :=\left(\bar{\partial}_{x_{1}} U(x), \ldots, \bar{\partial}_{x_{d}} U(x)\right)^{\top} \\
& \bar{\nabla} U(x) \in\left(\operatorname{Sym}_{n}(\mathbb{R})\right)^{d}\end{aligned}$ \\
\hline $\begin{array}{l}\text { structure } \\
\text { tensor }\end{array}$ & $G_{\rho} *\left(\bar{\nabla} u(x) \cdot(\bar{\nabla} u(x))^{\top}\right)$ & $\overline{\mathcal{S}}_{L}(U(x)):=G_{\rho} *\left(\bar{\nabla} U(x) \cdot(\bar{\nabla} U(x))^{\top}\right)$ \\
\hline product & $a \cdot b$ & $A \bullet_{J} B:=\frac{1}{2}(A B+B A)$ \\
\hline
\end{tabular}

Table 1: Extensions of elements of scalar valued calculus to the matrix-valued setting.

\section{The Full Structure Tensor $\overline{\mathcal{S}}_{L}$ for Matrix Fields}

\subsection{Derivation of $\overline{\mathcal{S}}_{L}$}

With the terminology introduced above we infer for the directional derivative of $x \mapsto U(x)$ in direction $v \in S^{d-1}$ :

$$
\begin{aligned}
\bar{\partial}_{v} U(x) & :=\left.\frac{d}{d h} U(x+h \cdot v)\right|_{h=0}=\sum_{k=1}^{d}\left(\partial_{x_{k}} u_{i j}(x)\right) v_{k} \\
& =(\bar{\nabla} U(x))^{\top}\left(\begin{array}{c}
v_{1} I_{n} \\
\vdots \\
v_{d} I_{n}
\end{array}\right) \\
& =v_{d} \bar{\partial}_{x_{1}} U(x)+\cdots+v_{d} \bar{\partial}_{x_{d}} U(x) \in \operatorname{Sym}_{n}(\mathbb{R})
\end{aligned}
$$

This expression is a symmetric matrix but aside from that in complete analogy to the corresponding real-valued term in the scalar case. In the scalar 
setting the direction of steepest descent/ascent would be given by the direction that optimises the directional derivative. However, in the matrix valued setting the entries of the generalised gradient are matrices, and finding an optimal unit vector $v$ that optimises the matrix-valued directional derivative is hindered by practical as well as theoretical obstacles: An optimisation relies on the presence of a total ordering, but on $\operatorname{Sym}_{n}(\mathbb{R})$ only partial ordering relations do exist. And even after choosing a partial ordering, e.g. the Loewner ordering [9], it is not clear how to obtain the optimal $v$ in a reasonable computational effort. A remedy for this difficulty is the projection of the set of symmetric matrices $\operatorname{Sym}_{n}(\mathbb{R})$ onto the real numbers by a so-called linear form. Each linear form on $\operatorname{Sym}_{n}(\mathbb{R})$ is of the form of a scalar product,

$$
M \longmapsto\langle A, M\rangle:=\operatorname{tr}_{A}(M):=\operatorname{tr}(A \cdot M)
$$

with a matrix $A \in \operatorname{Sym}_{n}(\mathbb{R})$, see [16]. Then one simply has

$$
\begin{aligned}
& \operatorname{argmax}\left\{\operatorname{tr}_{A}\left(\bar{\partial}_{v} U(x)\right) \mid\|v\|=1\right\}= \\
& \quad \frac{1}{\sqrt{\sum_{1}^{d}\left(\operatorname{tr}_{A}\left(\bar{\partial}_{x_{i}} U(x)\right)\right)^{2}}}\left(\operatorname{tr}_{A}\left(\bar{\partial}_{x_{1}} U(x)\right), \ldots, \operatorname{tr}_{A}\left(\bar{\partial}_{x_{d}} U(x)\right)\right) .
\end{aligned}
$$

We write $\operatorname{tr}=\operatorname{tr}_{I}$. Depending on the choice of $A$ we obtain the direction of strongest change at one point $x$ in the matrix field, or which boils down to the same thing: The strongest change in the scalar image $x \longmapsto \operatorname{tr}_{A}(U(x))$. Apparently this approach suffers from the same weakness as the direct direction estimation in scalar images, the danger of cancellation through averaging. This reveals the need for a structure tensor for matrix fields and also its basic construction principle. It is close at hand to define a structure tensor for matrix fields as follows:

$\overline{\mathcal{S}}_{L}(U(x)):=G_{\rho} *\left(\bar{\nabla} U(x) \cdot(\bar{\nabla} U(x))^{\top}\right)=\left(G_{\rho} *\left(\bar{\partial}_{x_{i}} U(x) \cdot \bar{\partial}_{x_{j}} U(x)\right)\right)_{i, j=1, \ldots, d}$

Here $G_{\rho} *$ indicate a convolution with a Gaussian of standard deviation $\rho$ or, more general, another appropriate averaging procedure. The parameter $\rho$ is suppressed to avoid notational clutter. We list some immediate properties of this construct:

1. $\overline{\mathcal{S}}_{L}(U(x))$ is a symmetric $n d \times n d$-block matrix with $d^{2}$ blocks of size $n \times n, \overline{\mathcal{S}}_{L}(U(x)) \in \operatorname{Sym}_{d}\left(\operatorname{Sym}_{n}(\mathbb{R})\right)=\operatorname{Sym}_{n d}(\mathbb{R})$. Typically for the 3D medical DT-MRI data one has $d=3$ and $n=3$, yielding a $9 \times 9$-matrix $\overline{\mathcal{S}}_{L}$. The symmetry follows from the fact that for all $i, j=1, \ldots, d$

$$
\begin{aligned}
\left(\bar{\partial}_{x_{i}} U(x) \cdot \bar{\partial}_{x_{j}} U(x)\right)^{\top} & =\left(\bar{\partial}_{x_{j}} U(x)\right)^{\top} \cdot\left(\bar{\partial}_{x_{i}} U(x)\right)^{\top} \\
& =\bar{\partial}_{x_{j}} U(x) \cdot \bar{\partial}_{x_{i}} U(x) .
\end{aligned}
$$


2. The structure tensor $\overline{\mathcal{S}}_{L}$ can be diagonalised as

$$
\overline{\mathcal{S}}_{L}(U)=\sum_{k=1}^{n d} \rho_{k} w_{k} w_{k}^{\top}
$$

with real eigenvalues $\lambda_{k}$ (w.l.o.g. arranged in decreasing order) and an orthonormal basis $\left\{v_{k}\right\}_{i=1, \ldots, n d}$ of $\mathbb{R}^{n d}$.

The usefulness of this construction will rise and fall with a positive answer to the following question:

How can we extract useful d-dimensional directional information from this full structure tensor $\overline{\mathcal{S}}_{L}$ ?

In the case of a structure tensor $S \in \operatorname{Sym}_{d}^{+}(\mathbb{R})$ for a scalar image its eigenvectors provide all the important directional information of the image. However, in the matrix-valued setting the eigenvectors $v_{i}$ of $\overline{\mathcal{S}}_{L}(U)$ are $n d$-dimensional vectors and they lack immediate physical interpretation. Hence, as such they do not provide really useful $d$-dimensional directional information. Instead, let us find an analog to the Rayleigh quotient of $S$

$$
\operatorname{argmax}\left\{v^{\top}\left(G * \nabla u \nabla u^{\top}\right) v \mid v \in \mathbb{R}^{d},\|v\|=1\right\}
$$

in the matrix field framework. We consider

$$
\begin{gathered}
\left(v_{1} I_{n} \cdots v_{d} I_{n}\right) \overline{\mathcal{S}}_{L}(U(x))\left(\begin{array}{c}
v_{1} I_{n} \\
\vdots \\
v_{d} I_{n}
\end{array}\right) \\
=\left(\begin{array}{ccc}
v_{1}^{2} G_{\rho} *\left(\bar{\partial}_{x_{1}} U\right)^{2} & \cdots & v_{1} v_{2} G_{\rho} *\left(\bar{\partial}_{x_{1}} U \cdot \bar{\partial}_{x_{d}} U\right) \\
\vdots & \ddots & \vdots \\
v_{1} v_{d} G_{\rho} *\left(\bar{\partial}_{x_{d}} U \cdot \bar{\partial}_{x_{1}} U\right) & \cdots & v_{d}^{2} G_{\rho} *\left(\bar{\partial}_{x_{d}} U\right)^{2}
\end{array}\right)
\end{gathered}
$$

Now we have to choose the real numbers $v_{1}, \ldots, v_{d}$ in an optimal way. Again we are facing difficulties caused by the absence of total ordering for matrices. In the case of a scalar image $u$ (a matrix field with $1 \times 1$-matrices) the optimal $v$ is the unit eigenvector corresponding to the largest eigenvalue of the structure tensor $S$. It will turn out to be convenient to utilise the notion of operator matrix.

The idea is to reduce $\overline{\mathcal{S}}_{L}(U) \in \operatorname{Sym}_{n d}(\mathbb{R})$ to a structure tensor $S(U) \in$ $\operatorname{Sym}_{n}(\mathbb{R})$ in a generalised projection step employing the block operator matrix

$$
\operatorname{Tr}_{A}:=\left(\begin{array}{ccc}
\operatorname{tr}_{A} & \cdots & 0 \\
\vdots & \ddots & \vdots \\
0 & \cdots & \operatorname{tr}_{A}
\end{array}\right)
$$


containing the trace operation. Again we set $\operatorname{Tr}:=\operatorname{Tr}_{I}$. This operator matrix acts on elements of the space $\left(\operatorname{Sym}_{n}(\mathbb{R})\right)^{d}$ as well as on block matrices via formal block-wise matrix multiplication,

$$
\left(\begin{array}{ccc}
\operatorname{tr}_{A} & \cdots & 0 \\
\vdots & \ddots & \vdots \\
0 & \cdots & \operatorname{tr}_{A}
\end{array}\right)\left(\begin{array}{ccc}
M_{11} & \cdots & M_{1 n} \\
\vdots & \ddots & \vdots \\
M_{n 1} & \cdots & M_{n n}
\end{array}\right)=\left(\begin{array}{ccc}
\operatorname{tr}_{A}\left(M_{11}\right) & \cdots & \operatorname{tr}_{A}\left(M_{1 n}\right) \\
\vdots & \ddots & \vdots \\
\operatorname{tr}_{A}\left(M_{n 1}\right) & \cdots & \operatorname{tr}_{A}\left(M_{n n}\right)
\end{array}\right)
$$

provided that the square blocks $M_{i j}$ are compatible with $\operatorname{tr}_{A}$, that means here, have the same size as $A$. The reason for choosing $\operatorname{tr}_{A}$ as reduction operators is their homogeneity:

$$
\operatorname{tr}_{A}(t M)=t \operatorname{tr}_{A}(M) \quad \text { for all } t \in \mathbb{R} .
$$

Note that $A=I$ provides the leading example since

$$
\begin{aligned}
\left(\begin{array}{ccc}
\operatorname{tr}_{A} & \cdots & 0 \\
\vdots & \ddots & \vdots \\
0 & \cdots & \operatorname{tr}_{A}
\end{array}\right)\left(\begin{array}{ccc}
M_{11} & \cdots & M_{1 d} \\
\vdots & \ddots & \vdots \\
M_{d 1} & \cdots & M_{d d}
\end{array}\right) \\
=\left(\begin{array}{ccc}
\operatorname{tr} & \cdots & 0 \\
\vdots & \ddots & \vdots \\
0 & \cdots & \operatorname{tr}
\end{array}\right)\left(\begin{array}{ccc}
A & \cdots & 0 \\
\vdots & \ddots & \vdots \\
0 & \cdots & A
\end{array}\right)\left(\begin{array}{ccc}
M_{11} & \cdots & M_{1 d} \\
\vdots & \ddots & \vdots \\
M_{d 1} & \cdots & M_{d d}
\end{array}\right)
\end{aligned}
$$

The subsequent result gives a first insight into the role of this reduction operation and its connection to other structure tensors:

Proposition: (The Standard Tensor as an Elementary Reduction of $\overline{\mathcal{S}}_{L}$ ) Let $U(x) \in \operatorname{Sym}_{n}(\mathbb{R})$ be a $d$-dimensional matrix-field. Then the standard tensor $J_{\rho}$ is a reduced version of the full tensor $\overline{\mathcal{S}}_{L}$,

$$
\operatorname{Tr} \overline{S_{L}}(U)=J_{\rho}(U) \in \operatorname{Sym}_{n}(\mathbb{R}) .
$$

Proof: First we realise that we can disregard the convolution with a Gaussian $G_{\rho}$ with integration scale $\rho$ or any other linear averaging process since the trace operation commutes with such linear mappings. Therefor we can deal with the partial derivatives $\partial_{x_{i}} u_{i, j}$ of the matrix components directly. Fix $p, q \in\{1, \ldots, d\}$. Then the $(p, q)$ th component of the standard structure tensor is given by

$$
J_{\rho}(U)=\sum_{i, j}^{d} \partial_{x_{p}} u_{i, j} \cdot \partial_{x_{q}} u_{i, j}
$$


However, we obtain for the $(p, q)$ th component of the reduced version of the full structure tensor $S_{L}(U)$

$$
\begin{aligned}
\operatorname{tr}\left(\bar{\partial}_{x_{p}} U \bar{\partial}_{x_{q}} U\right)=\sum_{i=1}^{d}\left(\bar{\partial}_{x_{p}} U \bar{\partial}_{x_{q}} U\right)_{i, i} & =\sum_{i=1}^{d} \sum_{j=1}^{d}\left(\partial_{x_{p}} u_{i, j} \cdot \partial_{x_{q}} u_{j, i}\right) \\
& =\sum_{i=1}^{d} \sum_{j=1}^{d}\left(\partial_{x_{p}} u_{i, j} \cdot \partial_{x_{q}} u_{j, i}\right),
\end{aligned}
$$

where the symmetry of the matrix $U$ accounts for the last equality. This proves the assertion.

The reduction operation is accompanied by an extension operation defined via the Kronecker product:

Definition: (Extension via Kronecker product)

The $I_{n}$-extension operation is the mapping from $\operatorname{Sym}_{d}(\mathbb{R})$ to $\operatorname{Sym}_{n d}(\mathbb{R})$ given by the Kronecker product $\otimes$ :

$$
\begin{aligned}
\left(\begin{array}{ccc}
v_{11} & \cdots & v_{1 d} \\
\vdots & \ddots & \vdots \\
v_{d 1} & \cdot & v_{d d}
\end{array}\right) \longmapsto & \left(\begin{array}{ccc}
v_{11} & \cdots & v_{1 d} \\
\vdots & \ddots & \vdots \\
v_{d 1} & \cdots & v_{d d}
\end{array}\right) \otimes\left(\begin{array}{ccc}
I_{n} & \cdots & I_{n} \\
\vdots & \ddots & \vdots \\
I_{n} & \cdots & I_{n}
\end{array}\right) \\
& :=\left(\begin{array}{ccc}
v_{11} I_{n} & \cdots & v_{1 d} I_{n} \\
\vdots & \ddots & \vdots \\
v_{d 1} I_{n} & \cdots & v_{d d} I_{n}
\end{array}\right) \cdot
\end{aligned}
$$

If the $d \times d$-matrix $\left(v_{i j}\right)_{i j}$ is Kronecker-multiplied with

$$
\left(\begin{array}{ccc}
C & \cdots & 0 \\
\vdots & \ddots & \vdots \\
0 & \cdots & C
\end{array}\right)\left(\begin{array}{ccc}
I_{n} & \cdots & I_{n} \\
\vdots & \ddots & \vdots \\
I_{n} & \cdots & I_{n}
\end{array}\right)=\left(\begin{array}{ccc}
C & \cdots & C \\
\vdots & \ddots & \vdots \\
C & \cdots & C
\end{array}\right)
$$

we speak of a $C$-extension.

\subsection{A novel diffusion tensor $\bar{D}$ for matrix fields}

Now it is possible to give an analog $\bar{D}$ to the diffusion tensor $D$ in the framework of matrix fields. We proceed in four steps:

1. The matrix field $\mathbb{R}^{d} \ni x \mapsto U(x)$ provides us with a module field of generalised gradients $\bar{\nabla} U(x)$ from which we construct the generalised structure tensor $\overline{\mathcal{S}}_{L} U(x)$ possibly with a certain integration scale $\rho$. This step corresponds exactly to the scalar case. 
2. We infer $d$-dimensional directional information by reducing $\overline{\mathcal{S}}_{L} U(x)$ with $\operatorname{tr}_{A}$ with the help of the block operator matrix given in (4) leading to a symmetric $d \times d$-matrix $S$, for example $S=J_{\rho}$ if $A=I_{n}$,

$$
S:=\left(\begin{array}{ccc}
\operatorname{tr}_{A} & \cdots & 0 \\
\vdots & \ddots & \vdots \\
0 & \cdots & \operatorname{tr}_{A}
\end{array}\right) \overline{\mathcal{S}}_{L} U(x)
$$

3. The symmetric $d \times d$-matrix $S$ is spectrally decomposed, and the tensor transfer map $H$ is applied to $S$ yielding the diffusion tensor $D$,

$$
D:=H(S)
$$

4. Finally we enlarge the $d \times d$-matrix $D$ to a $n d \times n d$-matrix $\bar{D}$ by the extension operation:

$$
\bar{D}=D \otimes\left(\left(\begin{array}{ccc}
C & \cdots & 0 \\
\vdots & \ddots & \vdots \\
0 & \cdots & C
\end{array}\right)\left(\begin{array}{ccc}
I_{n} & \cdots & I_{n} \\
\vdots & \ddots & \vdots \\
I_{n} & \cdots & I_{n}
\end{array}\right)\right)
$$

This last step gives another possibility to steer the filter process by the choice of the matrix $C$. However, this is the subject of current research. For this work we restricted ourselves to $C=I_{n}$.

\section{Coherence-Enhancing Diffusion Filtering for Matrix Fields}

Now we have gathered the necessary ingredients to formulate the matrixvalued equivalent to the scalar coherence enhancing diffusion as expressed in equation (1):

$$
\begin{aligned}
\bar{\partial}_{t} U-\sum_{i=1}^{d} \bar{\partial}_{x_{i}}(\bar{D} \bullet \bar{\nabla} U) & =0 \quad \text { in } I \times \Omega, \\
\bar{\partial}_{n} U & =0 \quad \text { in } I \times \partial \Omega, \\
U(x, 0) & =F(x) \quad \text { in } \Omega .
\end{aligned}
$$

Note that the Jordan-multiplication in $\bar{D} \bullet \bar{\nabla} U$ is understood in the blockwise sense of partitioned matrices. Moreover, we translated the divergence differential operator div $u=\sum_{i=1}^{d} \partial_{x_{i}} u$ acting on a vector-valued function 
$u=\left(u_{1}, \ldots, u_{d}\right)$ into its matrix-valued counterpart acting on a module field $W \in \operatorname{Sym}_{n}(\mathbb{R})^{d}$ by

$$
\overline{\operatorname{div}} W=\sum_{i=1}^{d} \bar{\partial}_{x_{i}} W .
$$

The numerical algorithm is inspired by the explicit scheme in [24]. We used a matrix-valued version employing the calculus framework for matrix fields as presented before. With a typical time step size of $\tau=\frac{1}{10}$ we observed numerical stability throughout our experiments.

\section{Experiments}

We use two data sets in our numerical experiments: The artificial matrix fields of $3 \times 3$-symmetric matrices exhibit various coherent structures ranging from simple line-like to curved features, see figures $1-4$. We will use these data to demonstrate the gap-closing, denoising, and enhancing properties of our technique. The other matrix fields stems from a 2-D slice extracted from a 3 -D DT-MRI data set of size of a $128 \times 128 \times 30$ of a human head, see Fig. 5 .

The data are represented as ellipsoids via the level sets of the quadratic form $\left\{x^{\top} A^{-2} x=\right.$ const. : $\left.x \in \mathbb{R}^{3}\right\}$ associated with a matrix $A \in \operatorname{Sym}^{+}(3)$. By using $A^{-2}$ the length of the semi-axes of the ellipsoid correspond directly with the three eigenvalues of the matrix. We have added random positive definite matrices to the data to demonstrate the denoising capabilities of our CEDfiltering concept. The eigenvectors of this noise were obtained by choosing Gaussian-distributed numbers with standard deviation $\sigma=1000$ and taking the absolute value for positive semidefiniteness. The high standard deviation can be explained by the fact that in real-world data the typical eigenvalues are in the order of magnitude of 1000. The eigenvectors of the artificial noise result in choosing three uniformly distributed angles and rotating the matrix by these angles around the coordinate axes. The resulting data is shown in Fig. 2.

The artificial data set displayed in Fig. 1 imitates a crossing of nerve fibers. Depending on the choice of the reduction matrix $A$ in $\operatorname{tr}_{A}$ either the diagonal directed downward, $A=\left(\begin{array}{rr}1 & -1 \\ -1 & 1\end{array}\right)$, or the one directed upward, $A=\left(\begin{array}{ll}1 & 1 \\ 1 & 1\end{array}\right)$, is given preference in the CED-filtering results. If no priority is set, $A=I$, a homogeneous structure is developing in the center, as it is expected due to the high symmetry of the image, see Fig. 1(d).

An impression of the denoising and gap-closing capabilities can be obtained 
from the results in Fig. 2. The noisy version of an artificial data set with a grid-like is CED-filtered. As before the directional preferences are conveyed by the reduction matrices $A=\left(\begin{array}{ll}1 & 0 \\ 0 & 0\end{array}\right)$ for the $x$-direction and $A=\left(\begin{array}{ll}0 & 0 \\ 0 & 1\end{array}\right)$ for the $y$-direction. The noise is removed and the lines in the selected direction are getting completed.

Fig. 3 shows the results of directionally selective CED-filtering if the direction of the coherent structure (here the $y$-direction) does not coincide with the direction of the ellipsoids (here the $x$-direction). By selecting the $x$-direction with a proper choice of $A$ we allow for the enhancement of coherent structures, that is, the closing of lines if there is a change in this $x$-direction. In Fig. 3(b) we have such a change, hence the present lines in $y$-direction are completed. We do not have a change in $y$-direction (the balls and ellipsoids have the same $y$-extension), hence the selection of the $y$-direction via $A=\left(\begin{array}{ll}0 & 0 \\ 0 & 1\end{array}\right)$ triggers no enhancement at all. Therefore this directional CED-filtering has no effect, see Fig. 3(c).

The experiment depicted in figure 4 demonstrates that even areas with no information may constitute a coherent structure that is preserved by CEDfiltering without directional preferences. The (almost) empty lines in $x$ direction are getting filled while the two lines in $y$-direction remain untouched by the filtering. The explanation is that in $x$-direction we have changes in the shape and orientation of the ellipsoids in the vicinity of the empty lines, hence, the gap-closing quality of CED-filtering comes into effect. However, proceeding in $y$ direction no changes of the surrounding ellipsoids is discernible, rendering the CED-filtering idle in this direction.

We applied CED-filtering without directional preference to real DT-MRI data as well in order to investigate its usefulness as a pre-processing step, e.g. for fiber tracking. The results are displayed in Figure 5 confirming the regularising effect and the enhancement of coherent structures such as the fiber bundles below the Corpus Callosum.

\section{Summary and Future Work}

In this chapter we have developed a novel structure tensor concept for matrix fields. This approach is based on an operator-algebraic view on matrices and their rich algebraic properties. We have shown how to infer directional information from this high dimensional data by specifying directional preferences, and clarified the standard structure tensor for matrix fields as a special case of our extended concept. An application of the new tensor concept enabled us to develop a directionally selective coherence-enhancing diffusion filtering 

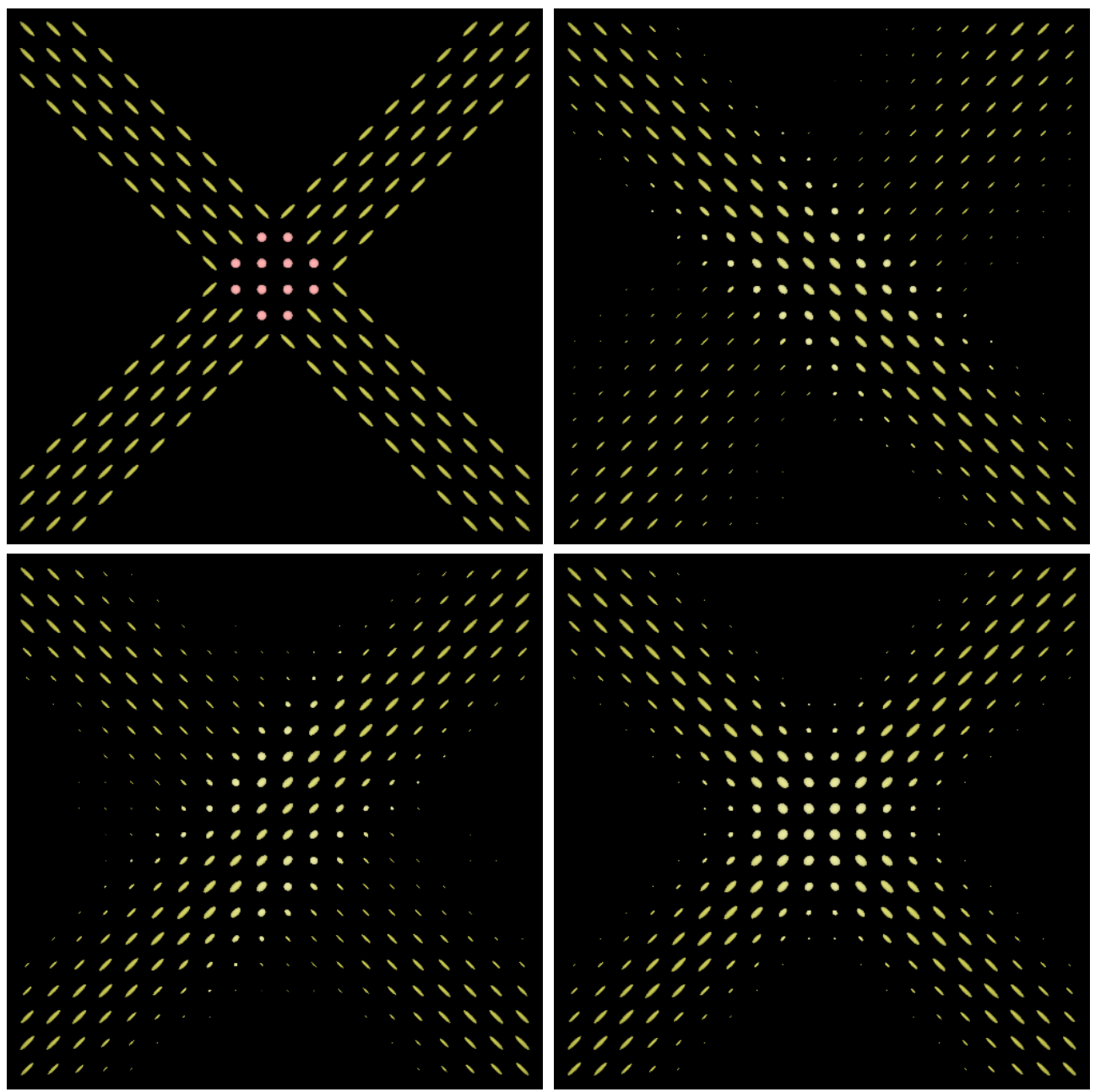

Figure 1: (a) Top left: Artificial data set of ellipsoids indicating a crossing. (b) Top right: Effect of coherence-enhancing filtering if preference is given to the direction $(1,-1)$ (c) Bottom left: Effect of coherence-enhancing filtering if preference is given to the direction (1,1). (d) Bottom right: Effect of coherence-enhancing filtering if no directional priority is established. 

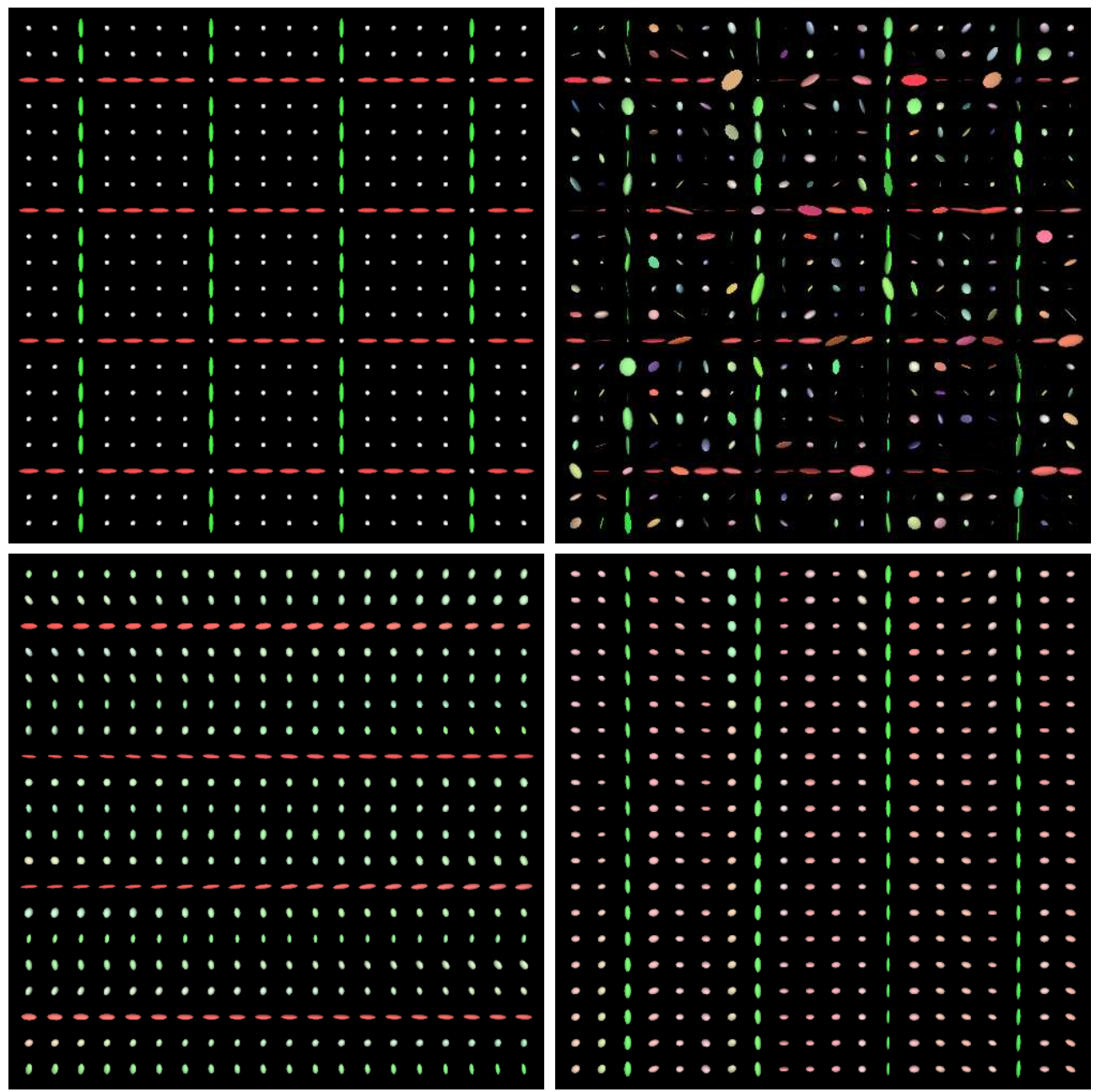

Figure 2: (a) Top left: Original matrix field with grid-like structure. (b) Top right: (a) polluted with truncated Gaussian noise in the eigenvalues while the orthogonal matrices result from three Euler matrices with uniformly distributed angles.

(c) Bottom left: Result of CED-filtering with preference on the horizontal x-direction. (d) Bottom right: The same but with preference on the vertical y-direction. 

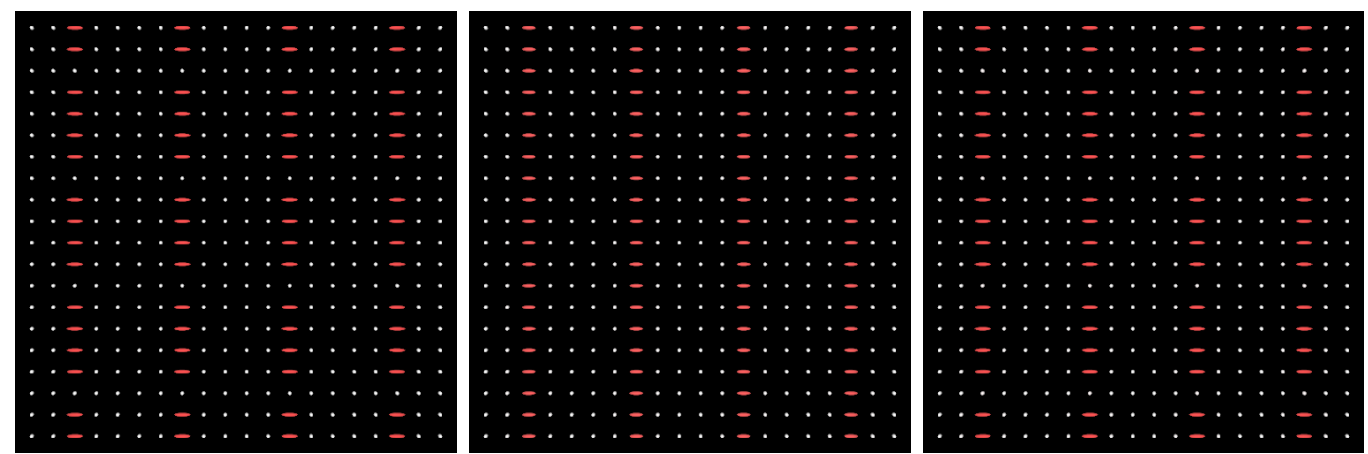

Figure 3: (a) Left: Original image with coherent structure in $y$-direction and ellipsoids pointing in $x$-direction. (b) Middle: Result of CED-filtering with preference on the horizontal x-direction. (c) Right: Result of CED-filtering with preference on the horizontal $y$-direction.
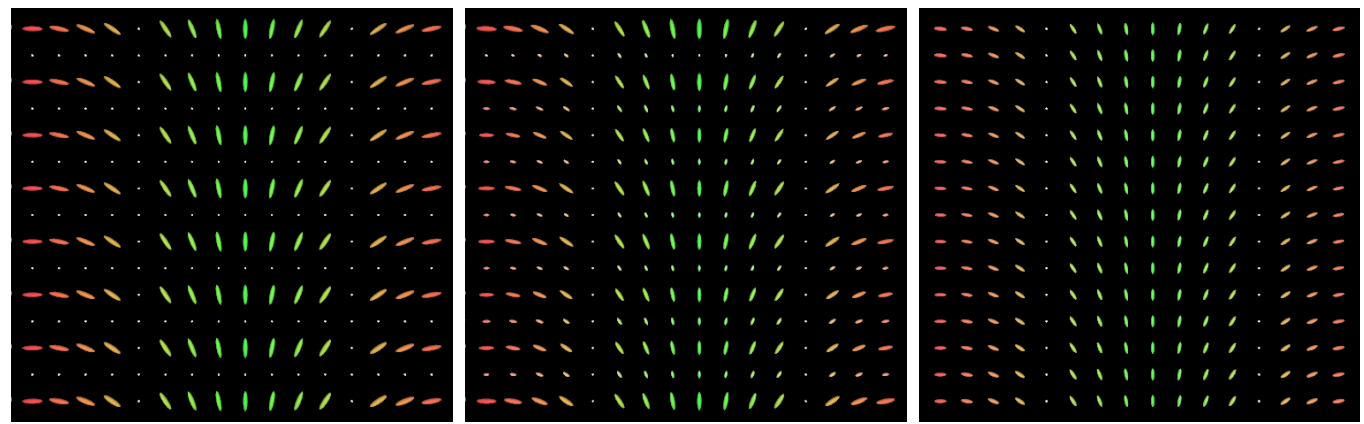

Figure 4: (a) Left: Artificial incomplete coherent structure. (b) Middle: After CED-filtering with stopping time $t=0.3$. (c) Right: After CEDfiltering with stopping time $t=3$ 

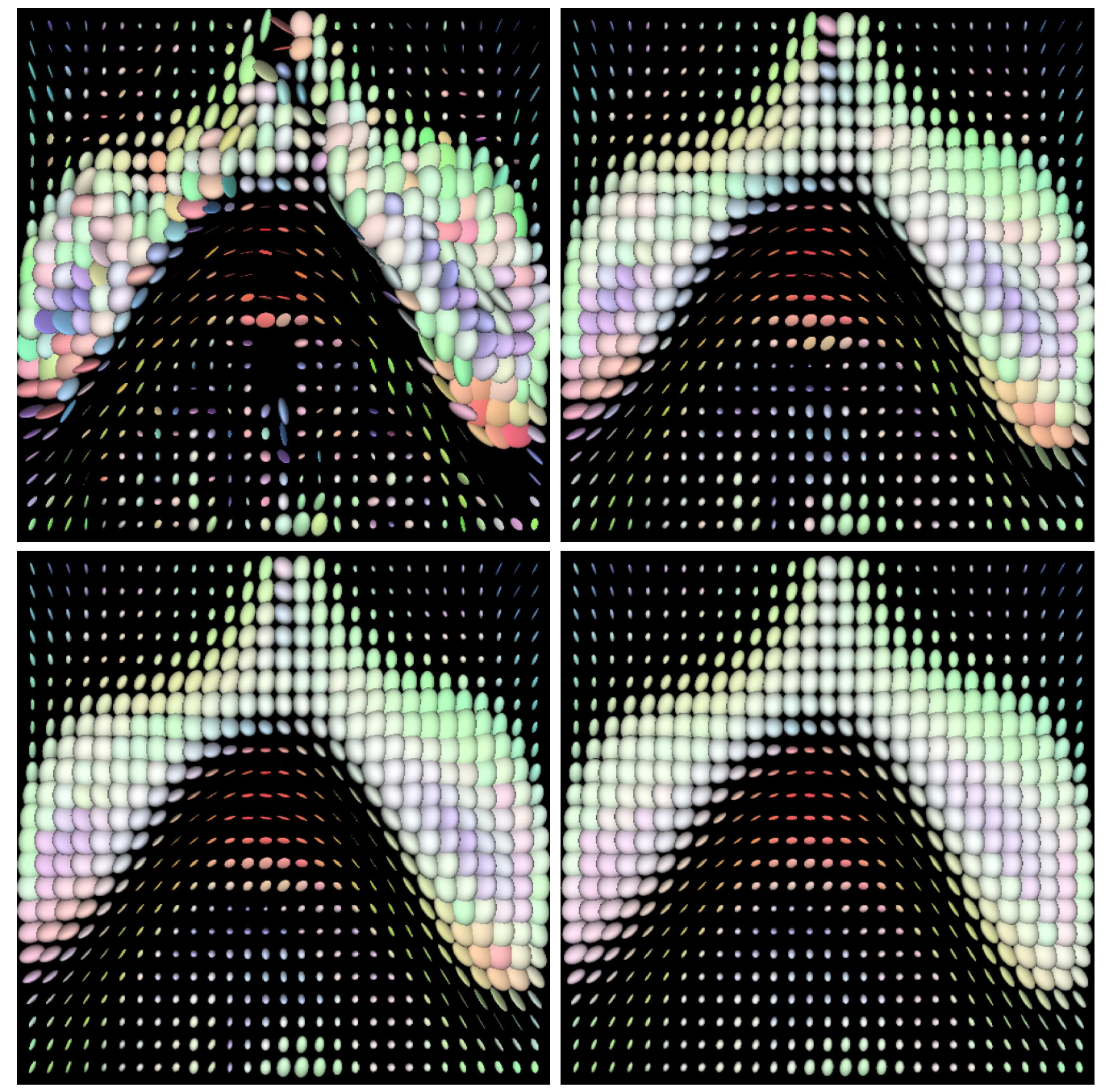

Figure 5: (a) Top left: Original DT-MRI data: lower part of the Corpus Callosum fiber area. (a) Top right: After CED-filtering with stopping time $t=1$. (b) Bottom left: CED-filtering with $t=3$. (c) Bottom right: CED-filtering with $t=5$. 
of matrix fields by employing a generic differential calculus framework for matrices. The matrix-valued CED-filtering exhibits similar behaviour as its scalar counterpart.

Current work encompasses the investigation of further opportunities to steer the filtering process,e.g. in the extension step, and its relation to other customisable tensor concepts for matrix fields. Future research will focus on further applications of the extended structure tensor concepts in image processing for matrix fields.

\section{Acknowledgments}

We are grateful to Anna Vilanova i Bartrolí (Eindhoven Institute of Technology) and Carola van Pul (Maxima Medical Center, Eindhoven) for providing us with the DT-MRI data set.

\section{References}

[1] V. Arsigny, P. Fillard, X. Pennec, and N. Ayache. Fast and simple calculus on tensors in the log-Euclidean framework. In J. Duncan and G. Gerig, editors, Medical Image Computing and Computer-Assisted Intervention - MICCAI 2005, Part I, volume 3749 of LNCS, pages 115122. Springer, 2005.

[2] V. Arsigny, P. Fillard, X. Pennec, and N. Ayache. Log-Euclidean metrics for fast and simple calculus on diffusion tensors. Magnetic Resonance in Medicine, 56(2):411-421, 2006.

[3] J. Bigun. Vision with Direction. Springer, Berlin, 2006.

[4] T. Brox and J. Weickert. A TV flow based local scale estimate and its application to texture discrimination. Journal of Visual Communication and Image Representation, 17(5):1053-1073, October 2006.

[5] T. Brox, J. Weickert, B. Burgeth, and P. Mrázek. Nonlinear structure tensors. Image and Vision Computing, 24(1):41-55, January 2006.

[6] B. Burgeth, A. Bruhn, S. Didas, J. Weickert, and M. Welk. Morphology for matrix-data: Ordering versus PDE-based approach. Image and Vision Computing, 25(4):496-511, 2007.

[7] B. Burgeth, S. Didas, L. Florack, and J. Weickert. A generic approach for singular PDEs for the processing of matrix fields. In F. Sgallari, 
F. Murli, and N. Paragios, editors, Scale Space and Variational Methods in Computer Vision, volume 4485 of Lecture Notes in Computer Science, pages 556-567. Springer, Berlin, 2007.

[8] B. Burgeth, S. Didas, L. Florack, and J. Weickert. A generic approach to diffusion filtering of matrix-fields. Computing, 81:179-197, 2007.

[9] B. Burgeth, N. Papenberg, A. Bruhn, M. Welk, C. Feddern, and J. Weickert. Morphology for higher-dimensional tensor data via Loewner ordering. In C. Ronse, L. Najman, and E. Decencière, editors, Mathematical Morphology: 40 Years On, volume 30 of Computational Imaging and Vision, pages 407-418. Springer, Dordrecht, 2005.

[10] C. Chefd'Hotel, D. Tschumperlé, R. Deriche, and O. Faugeras. Constrained flows of matrix-valued functions: Application to diffusion tensor regularization. In A. Heyden, G. Sparr, M. Nielsen, and P. Johansen, editors, Computer Vision - ECCV 2002, volume 2350 of Lecture Notes in Computer Science, pages 251-265. Springer, Berlin, 2002.

[11] O. Coulon, D. C. Alexander, and S. A. Arridge. A regularization scheme for diffusion tensor magnetic resonance images. In M. F. Insana and R. M. Leahy, editors, Information Processing in Medical Imaging - IPMI 2001, volume 2082 of Lecture Notes in Computer Science, pages 92-105. Springer, Berlin, 2001.

[12] S. Di Zenzo. A note on the gradient of a multi-image. Computer Vision, Graphics and Image Processing, 33:116-125, 1986.

[13] C. Feddern, J. Weickert, B. Burgeth, and M. Welk. Curvature-driven PDE methods for matrix-valued images. International Journal of Computer Vision, 69(1):91-103, August 2006.

[14] W. Förstner and E. Gülch. A fast operator for detection and precise location of distinct points, corners and centres of circular features. In Proc. ISPRS Intercommission Conference on Fast Processing of Photogrammetric Data, pages 281-305, Interlaken, Switzerland, June 1987.

[15] Y. Gur and N. Sochen. Diffusion over tensor fields via lie group pde flows. In M. Farber, R. Ghrist, M. Burger, and D. Koditschek, editors, Topology and Robotics, Contemporary Mathematics, pages 59-74. Springer, Berlin, 2007.

[16] R. A. Horn and C. R. Johnson. Matrix Analysis. Cambridge University Press, Cambridge, UK, 1990. 
[17] H. Knutsson. Representing local structure using tensors. In The 6th Scandinavian Conference on Image Analysis, pages 244-251, Oulu, Finland, June 1989. Report LiTH-ISY-I-1019, Computer Vision Laboratory, Linköping University, Sweden, 1989.

[18] C. Lenglet, M. Rousson, R. Deriche, and O. Faugeras. Statistics on the manifold of multivariate normal distributions: theory and application to diffusion tensor mri processing. Journal of Mathematical Imaging and Vision, 25(3):423-444, 2006.

[19] T. McGraw, B. C. Vemuri, Y. Chen, M. Rao, and T. H. Mareci. DTMRI denoising and neuronal fiber tracking. Medical Image Analysis, $8: 95-111,2004$.

[20] X. Pennec, P. Fillard, and N. Ayache. A riemannian framework for tensor computing. International Journal of Computer Vision, 66(1):4166,2006 .

[21] T. Schultz, B. Burgeth, and J. Weickert. Flexible segmentation and smoothing of DT-MRI fields through a customizable structure tensor. In G. Bebis, R. Boyle, B. Parvin, D. Koracin, P. Remagnino, A. Nefian, G. Meenakshisundaram, , V. Pascucci, J. Zara, J. Molineros, H. Theisel, and T. Malzbender, editors, Advances in Visual Computing, volume 4291 of Lecture Notes in Computer Science, pages 454-464. Springer, Berlin, 2006.

[22] D. Tschumperlé and R. Deriche. Diffusion tensor regularization with constraints preservation. In Proc. 2001 IEEE Computer Society Conference on Computer Vision and Pattern Recognition, volume 1, pages 948-953, Kauai, HI, December 2001. IEEE Computer Society Press.

[23] D. Tschumperlé and R. Deriche. Orthonormal vector sets regularization with PDE's and applications. International Journal of Computer Vision, 50(3):237-252, December 2002.

[24] J. Weickert. Anisotropic Diffusion in Image Processing. Teubner, Stuttgart, 1998.

[25] J. Weickert. Coherence-enhancing diffusion filtering. International Journal of Computer Vision, 31(2/3):111-127, April 1999.

[26] J. Weickert. Coherence-enhancing diffusion of colour images. Image and Vision Computing, 17(3-4):199-210, March 1999. 
[27] J. Weickert and T. Brox. Diffusion and regularization of vector- and matrix-valued images. In M. Z. Nashed and O. Scherzer, editors, Inverse Problems, Image Analysis, and Medical Imaging, volume 313 of Contemporary Mathematics, pages 251-268. AMS, Providence, 2002.

[28] J. Weickert and H. Hagen, editors. Visualization and Processing of Tensor Fields. Springer, Berlin, 2006. 